\title{
Including spin in the Raychaudhuri equation
}

\author{
A. J. Fennelly \\ Teledyne Brown Engineering and the University of Alabama in Huntsville, Huntsville, Alabama 35899 \\ Jean P. Krisch \\ Department of Physics, University of Michigan, Ann Arbor, Michigan 48109 \\ John R. Ray \\ Department of Physics and Astronomy, Clemson University, Clemson South Carolina 29634 \\ Larry L. Smalley \\ Department of Physics, The University of Alabama in Huntsville, Huntsville, Alabama 35899
}

(Received 2 April 1990; accepted for publication 18 July 1990)

The Raychaudhuri equation for a spin fluid matter content is developed. The equation is applied to the behavior of an irrotational, unaccelerated fiuid. The development of singularities in the expansion is studied for constant spin densities.

\section{INTRODUCTION}

The Raychaudhuri equation is a very useful tool in general relativity. It can be written as ${ }^{1}$

$$
\begin{gathered}
\dot{\theta}+\theta^{2} / 3-\dot{U}_{\cdot{ }^{\prime}}+\left(\sigma^{a l \prime} \sigma_{a h}-\Omega^{a t} \Omega_{u b}\right) \\
+(\kappa / 2)(\rho+3 p)=0,
\end{gathered}
$$

where $U^{a}$ is the fluid velocity, $\dot{U}^{a}$ the acceleration, $\theta$ the expansion, $\Omega_{a b}$ the fluid vorticity, and $\sigma_{a b}$ is the shear; $\rho$ is the fluid energy density, $p$ the pressure, and $\kappa=8 \pi G$.

The relationships this equation gives between fluid parameters and matter content have been the basis of some very interesting general theorems ${ }^{2}$ which can be applied to the development of singularities in the universe treated as a cosmological fluid.

The Raychaudhuri equation describes a perfect fluid with a stress energy tensor

$$
T_{a h}=(\rho+p) U_{a} U_{n}+p g_{a b} .
$$

There are other stress energy tensors that describe more complex fluids; for example, fluids with viscosity and heat transfer. In treating cosmological problems, parameters of this type are currently of limited interest. There is, however, one parameter, intrinsic spin, which could have a significant effect on cosmological Raychaudhuri arguments. The Raychaudhuri equation (1), does not include any stress energy content due to an intrinsic fluid angular momentum. Since the "galactic molecules" that make up the cosmological fluid do have intrinsic spin, it would be very interesting to properly examinc the effects of spin on problems treatable by the Raychaudhuri equation.

Previous authors have considered spin in the Raychaudhuri equation within the Einstein-Cartan extension of general relativity. ${ }^{3-5}$ These extensions have included spin by extending the normal Christoffel connection to include the torsional connection. In this paper we will consider a stress energy tensor developed by Ray and Smalley" that explicitly includes spin. It describes a perfect spin fluid, a fluid that has an intrinsic spin density $S_{u b}$ defined across its extent. We will derive the Raychaudhuri equation for spin fluids in general relativity and in the Einstein-Cartan theory. In the next section we briefly review the spin-fluid stress energy tensor. The Raychaudhuri equation is derived in Sec. III. In the last part of the paper we apply the spin-extended Raychaudhuri equation to the behavior of the expansion in irrotational, geodetic fluids.

\section{SPIN FLUIDS}

\section{A. Spin density}

The spin density $S_{a b}$ defines a density of angular momentum in the fluid. It is related to the trace free torsion ${ }^{7}$ $\bar{S}_{a b}{ }^{c}$ by

$$
\bar{S}_{a b}{ }^{c}=(\kappa / 2) S_{a b} U^{c} \text {. }
$$

The complete torsion $S_{a b}{ }^{c}$ related to the antisymmetric part of the connection,

$$
S_{a b}{ }^{c}=\Gamma_{[a b\}}^{c}
$$

is proportional to the trace free torsion plus a term involving a torsion vector,

$$
S_{a b}{ }^{c}=\bar{S}_{a b}{ }^{c}-\frac{2}{3} \delta_{\mid a}^{c} S_{b \mid x}{ }^{x} .
$$

The torsion vector $S_{h x}{ }^{x}$ is frequently written as $S_{b}$. It is not to be confused with the spin vector

$$
\bar{S}^{a}-\epsilon^{u \text { thcid }}\left(U_{b} / 2 \sqrt{g}\right) S_{c d} \text {. }
$$

The spin density $S_{c d}$ is constrained by the Frenkel condition $^{8}$

$$
U^{c} S_{c d}=0 .
$$

The torsion vector represents that part of the complete torsion that is not Frenkel constrained. There is some evidence that the torsion vector is useful in systems where particle number is nonconserved. ${ }^{7,9}$ Normally the torsion vector is not included in spin-fluid calculations. We include it for completeness. 


\section{B. Stress energy tensor}

The fluid vorticity $\Omega_{a b}$ is defined by

$$
\Omega_{a b}=-U_{\{a ; b\}}-\dot{U}_{\mid a} U_{b \mid} .
$$

In addition to the angular velocity described by this vorticity, there is an angular velocity $\omega_{a b}$, associated with an intrinsic spin density. The rotation associated with the spin can be described by the tensor

$$
\omega_{a b}=\dot{A}_{a}^{(\mu)} A_{b(\mu)},
$$

where $A_{(\mu)}^{a}$ is a metric based, orthonormal tetrad chosen so that $A_{(0)}^{a}$ lies along the fluid velocity $U^{a}$. Tetrad indices are in parentheses. $(\mu)=(0,1,2,3)$ and are raised and lowered with the Minkowski metric $(-+++)$.

The Ray-Smalley spin-fluid stress energy tensor can be written as the sum of two parts:

$$
T^{a b}=T_{(\text {fluid) }}^{a b}+T_{(\text {spin) }}^{a b},
$$

where $T_{\text {(fluid) }}^{a b}$ is the usual perfect fluid stress encrgy tensor described by Eq. (2).

The spin-fluid portion of the stress energy tensor is

$T_{\text {(spin) }}^{a b}=2 U^{(a} S^{b r c} \dot{U}_{c}+\nabla_{c}^{*}\left(U^{(a} S^{b) c}\right)-\omega^{c(a} S^{b)}{ }_{c}$.

This stress energy tensor is valid in both general relativity (GR) and in the Einstein-Cartan theory (ECT). In GR, the covariant derivative, $\nabla_{a}$ involves the usual metric conncction. In ECT, the covariant "star" derivative is written

$$
\nabla_{a}^{*}=\nabla_{a}+2 S_{a},
$$

where $\nabla_{a}$ contains a spin contribution in addition to the metric connection. $S_{a}$ is the torsion vector.

\section{THE RAYCHAUDHURI EQUATION}

\section{A. General relativity--Riemannian space-time}

The field equations in GR are

$$
R_{a b}=\kappa\left(T_{a b}-\frac{1}{2} g_{a b} T\right) \text {. }
$$

The Raychaudhuri equation can be derived from the field equations by projecting out the components along the fluid velocity:

$$
R_{a h} U^{a} U^{b}=\kappa\left(T_{a b} U^{a} U^{b}+T / 2\right) .
$$

The spin extended Raychaudhuri equation is

$$
\begin{gathered}
\dot{\theta}+\theta^{2} / 3-\dot{U}_{; a}^{a}+\left(\sigma^{a b} \sigma_{a b}-\Omega^{a b} \Omega_{a b}\right)-\kappa \Omega^{a b} S_{a b} \\
+(\kappa / 2) \omega^{a b} S_{a b}+(\kappa / 2)(p+3 p)=0 .
\end{gathered}
$$

The new contributions from the spin are both kinetic energy type terms.

\section{B. Einstein-Cartan-Riemann-Cartan space-times}

In the Einstein-Cartan theory, the metric field equations are ${ }^{10}$

$$
G_{u b}-\nabla_{c}^{*}\left(\bar{Q}_{a b}{ }^{c}-\bar{Q}_{b}{ }_{a}{ }+\bar{Q}^{c}{ }_{a b}\right)=\kappa T_{u b},
$$

where the modified torsion "is

$$
\bar{Q}_{a b}{ }^{c}=S_{a b}{ }^{c}+2 \delta_{\mid a}{ }^{c} S_{b\rangle} \text {. }
$$

Separating the left-hand side of Eq. (16) (Ref. 12) into the GR Ricci tensor plus torsion contributions we obtain
$R_{u b}($ GR $)+2 S_{u c}{ }^{\prime \prime} S_{b l}{ }^{\prime \prime}+2 S_{u c}{ }^{d} S_{b d}{ }^{c}-S^{c d t}{ }_{u} S_{c b b}-4 S_{u p} S_{b}$

$$
=\kappa\left(T_{a b}-\frac{1}{2} g_{a b} T\right) \text {. }
$$

Contracting Eq. (18) with $U^{a} U^{b}$, we obtain the Raychaudhuri equation in a Riemann-Cartan space-time:

$$
\begin{aligned}
\dot{\theta}+ & \frac{\theta^{2}}{3}-\dot{U}_{s t}^{u}+\sigma^{u t h} \sigma_{u t h}-\left(\Omega^{u t h}+\frac{\kappa}{2} S^{a t h}\right)\left(\Omega_{a t h}+\frac{\kappa}{2} S_{u t h}\right) \\
& +\frac{\kappa}{2}\left(\omega_{u t h}-\frac{\kappa}{2} S_{a t}\right) S^{u t h} \\
& -\frac{8}{3}\left(S^{a} U_{a t}\right)^{2}+\frac{\kappa}{2}[\rho+3 p]=0
\end{aligned}
$$

It is quite different from its GR counterpart and also from other treatments that did not include spin in the stress encrgy content. In Eq. (19), all covariant derivatives contain only Christoffel connections. The spin connection has been separated from the covariant derivatives and included explicitly.

\section{EXPANSION IN IRROTATIONAL AND UNACCELERATED FLUIDS}

\section{A. General relativity}

If the vorticities and the acceleration are zero, the Raychaudhuri equation can be written

$$
\mathrm{GR}: \dot{\theta}+\theta^{2} / 3+\sigma^{a b} \sigma_{a b}=-(\kappa / 2)(\rho+3 p) .
$$

Spin does not enter the GR result.

The solution to Eq. (20) is well known: $:^{2,13}$

$$
\frac{1}{\theta(\tau)} \leqslant \frac{1}{\theta(0)}+\frac{\tau}{3}
$$

and predicts an expansion singularity if the expansion is initially converging.

\section{B. Einstein-Cartan}

In Einstein-Cartan theories, the vorticities normally have a spin contribution due to the torsional connection. This contribution has been separated out of Eq. (19). The full fluid vorticity $\bar{\Omega}_{t t}$, and tetrad rotation vector $\bar{\omega}_{u,}$, can be written as

$$
\begin{aligned}
& \bar{\omega}_{u t h}=\omega_{u t h}-k\left(S_{u t h} / 2\right), \\
& \bar{\Omega}_{a t h}=\Omega_{a t h}+k\left(S_{u t h} / 2\right) .
\end{aligned}
$$

For no acceleration, $\omega_{u t \prime}$ equals $\Omega_{u i t}$. There are two possible ways that irrotational could be interpreted. The first would be the strict zero vorticity condition

$$
\bar{\omega}_{d t}=\bar{\Omega}_{d t r}=0 .
$$

The resulting Raychaudhuri equation, with zero torsion vector, is identical to the GR result, Eq. (20). The second interpretation would zero the metric GR vorticities:

$$
\omega_{u t r}=\Omega_{u b}=0 \text {. }
$$

The Raychaudhuri equation for this case is

$$
\dot{\theta}+\frac{\theta^{2}}{3}+\sigma^{u t h} \sigma_{u t s}-\frac{\kappa^{2}}{2} S^{u l t} S_{u t s}+\frac{\kappa}{2}(\rho+3 p)=0 .
$$

Equation (26) leads to the expansion condition 


$$
\dot{\theta}+\theta^{2} / 3-\left(\kappa^{2} / 2\right) S^{2} \geq 0,
$$

where $S^{2}=S^{o \prime \prime} S_{u \prime \prime}$. It is clear that the presence of spin in the Einstein-Cartan treatment could influence the development of a congruence singularity.

The solution to (27) depends on the functional form of the spin that may depend on the metric solution. Assuming a constant spin density for purposes of discussion, we integrate to find

$$
\frac{\theta(t)-\sqrt{\frac{3}{2}} \kappa S}{\theta(t)+\sqrt{\frac{3}{2}} \kappa S} \geqslant\left(\frac{\theta(0)-\sqrt{\frac{3}{2}} \kappa S}{\theta(0)+\sqrt{\frac{3}{2}} \kappa S}\right) e^{2 \kappa S t / 16}
$$

or

$$
\theta(t) \geqslant \sqrt{\frac{3}{2}} \kappa S\left[\left(1+A e^{2 \kappa S t /{ }^{\circ}}\right) /\left(1-A e^{2 \kappa S t / 0}\right)\right],
$$

with

$$
\begin{aligned}
& A=\left[\theta(0)-\sqrt{\frac{3}{2}} \kappa S\right] /\left[\theta(0)+\sqrt{\frac{3}{2}} \kappa S\right], \\
& A \neq 1 .
\end{aligned}
$$

There are four separate cases to consider in discussing the development of singularities in the expansion function.

(1) $A>1$ : $A$ will be positive and greater than 1 if the expansion is converging

$$
\theta(0)<0 \text { and }|\theta(0)|>\kappa \sqrt{\frac{3}{2}} S .
$$

No singularities develop and the congruence remains converging.

(2) $0<A<1$ : Values of $A$ in this range are generated by a positive initial expansion with

$$
\theta(0)>\sqrt{\frac{3}{2}} \kappa S \text {. }
$$

A singularity will develop for this case.

(3) $A<0$ : Negative $A$ can occur if

$$
\theta(0)<0 \text { and }|\theta(0)|<\sqrt{\frac{3}{2}} \kappa S
$$

or

$$
\theta(0)>0 \text { and } \theta(0)<\sqrt{\frac{3}{2}} \kappa S .
$$

For negative $A$, the expansion remains well behaved.

(4) The conditions $\theta(0)= \pm \sqrt{\frac{3}{2}} \kappa S$ lead to a constant expansion.

\section{DISCUSSION}

We have extended the perfect-fluid Raychaudhuri equation to include intrinsic spin and applied the results to the behavior of the expansion parameter in an irrotational, geo- detic fluid. This particular application was chosen because the development of space-time singularities is sometimes implied by the development of singularities in the expansion parameter of a congruence. ${ }^{13}$ We have shown that the presence of spin in Riemann-Cartan space-times can avert the development of an expansion singularity. The complete implications of this result for the development of metric singularities in space-times with torsion is under investigation.

Previous work on the development of singularities has considered the effect of nuclear spins in the ultra high matter density conditions just before a final collapse. ${ }^{11}$ Although a continuium of nuclear spins is a spin fluid, our formulation also includes the macroscopic view of spin density as described by Kopczynski, ${ }^{14}$ Bailey, ${ }^{15}$ Israel, ${ }^{16}$ and Israel and Bailey. ${ }^{17}$ In this picture, continuous media may have internal spin densities and collections of objects such as protogalaxies, turbulent eddies, or (primeval) black holes may be described as a spin fluid.

Other possible applications of the spin extended Raychaudhuri equation are star or galaxy formation from the collapse of matter clouds with intrinsic spin density, as well as the inflationery formation or collapse of universes with spin. The inclusion of spin in the Raychaudhuri equation should lead to many interesting and valuable results.

${ }^{1}$ A. K. Raychaudhuri, Theoretical Cosmology (Clarendon, Oxford. 1979), p. 81 .

${ }^{2}$ Reference 1, p. 80

${ }^{3}$ J. Tafel, Phys. Lett. A 45, 341 (1973).

${ }^{4} \mathrm{~J}$. Steward and P. Hajicek, Nature Phys. Sci. 244, 96 (1973).

${ }^{5}$ F. W. Hehl, GRG 4, 333 (1973); 5, 491 (1974).

'J. R. Ray and L. L. Smalley, Phys. Rev. D 26, 2619 (1982); Phys. Rev. D 27, 1383 (1983); Phys. Rev. Lett. 49, 1059 (1987); Phys. Rev. Lett. 50, 626E (1983); Phys. Rev. D 26, 2615 (1982).

${ }^{7}$ L. L. Smalley and J. R. Ray, GRG 18, 549 (1986).

${ }^{8}$ J. Frenkel, Z. Phys. 37, 243 (1926).

${ }^{9}$ L. D. Landau and E. M. Lifshitz, Fluid Mechanics (Addison-Wesley, Reading, MA, 1959), p. 501.

${ }^{10}$ V. G. Krechet, Izvest. Fiz. 12, 9 (1985).

"F. W. Hehl, P. von der Hyde, and G. D. Kerluk, Phys. Rev, D 10, 1066 (1974).

${ }^{12}$ A. K. Raychaudhuri, Theoretical Cosmology (Clarendon, Oxford, 1979), p. 185.

${ }^{13}$ R. M. Wald, General Relativity (University of Chicago, Chicago, IL, 1984), p. 220.

${ }^{14}$ W. Kopczynski, Phys. Lett. A 39, 219 (1977).

${ }^{15}$ I. Bailey, Ann. Phys. (NY) 119, 76 (1979).

${ }^{10} \mathrm{~W}$. Israel, Lett. Nuovo Cimento 7, 860 (1973).

${ }^{17}$ I. Bailey and W. Israel, Commun. Math. Phys. 42, 65 (1975). 